\title{
Tangence
}

\section{Visages montréalais de la marginalité québécoise dans l'œuvre de Michel Tremblay}

\section{Alain-Michel Rocheleau}

Numéro 48, octobre 1995

Montréal et Vancouver : parcours urbains dans la littérature et le cinéma

URI : https://id.erudit.org/iderudit/025861ar

DOI : https://doi.org/10.7202/025861ar

Aller au sommaire du numéro

Éditeur(s)

Tangence

ISSN

0226-9554 (imprimé)

1710-0305 (numérique)

Découvrir la revue

Citer cet article

Rocheleau, A.-M. (1995). Visages montréalais de la marginalité québécoise dans l'œuvre de Michel Tremblay. Tangence, (48), 43-55.

https://doi.org/10.7202/025861ar d'utilisation que vous pouvez consulter en ligne. 


\section{Visages montréalais de la marginalité québécoise dans l'œuvre de Michel Tremblay}

\section{Alain-Michel Rocheleau}

Dans une œuvre littéraire, l'environnement des personnages prend une valeur des plus significatives. Miroir d'une réalité sociale, politique et culturelle qu'un auteur tend à illustrer dans ses écrits, l'espace reflète, bien souvent, les limites géographiques et idéologiques d'une société donnée, d'une ville ou d'un quartier, en apportant un éclairage particulier sur les sentiments et les valeurs des individus représentés dans la littérature.

Comme le démontre très bien Robert Mann, dans un article intitulé "L'image de Montréal dans les Cbroniques du Plateau Mont-Royal", l'illustration d'espaces différents, dans un texte littéraire, prend une valeur qui déborde la simple description d'un territoire rural ou urbain. Comparant la description de Montréal dans Bonbeur d'occasion de Gabrielle Roy et les Cbroniques $d u$ Plateau Mont-Royal de Michel Tremblay, l'auteur conclut ainsi son étude: "Entre le Saint-Henri de Gabrielle Roy et le Plateau Mont-Royal de Tremblay, il y a bien plus que l'espace de quelques rues [...] il y a bien plus même que le fossé d'une génération. Il y a deux points de vue totalement différents, l'un fait de grande pitié, d'indignations immédiates, l'autre de nostalgie. "1 Pour ma part, je me limiterai dans cet article à démontrer que les structures de certains espaces représentés dans l'œuvre de Tremblay sont corrélatives des structures sociales qui caractérisaient le Montréal des années quarante et cinquante et qui minimisaient l'importance de la culture francophone qualifiée, durant cette période, de "populaire". En vivant dans la métropole du Québec, où la culture anglophone semblait alors prédominer, les Montréalais de langue française ont dû composer avec cette réalité socio-culturelle qui leur apparaissait tantôt séduisante (au point d'amener un certain nombre de citoyens à s'identifier à la culture dominante - phénomène d'assimilation), tantôt mena-

1 Robert Mann, "L'image de Montréal dans les Cbroniques du Plateau MontRoyal", Études canadiennes, n 19, décembre 1985, p. 132. 
44

çante (forçant plusieurs d'entre eux à se protéger de cette culture - phénomène de "ségrégation volontaire"). Or, comme nous le verrons, ces deux phénomènes caractérisent l'attitude des marginaux présents dans l'œuvre de Tremblay. En partant de ces considérations, je tenterai de démontrer que la marginalité des protagonistes peut être considérée comme une métaphore de la société montréalaise d'alors.

Entre les rues Fabre et Saint-Laurent, les personnages de Tremblay forment un vaste chœur de gens du "petit peuple" et de laissés pour compte pour qui la réalité montréalaise n'est vécue qu'à travers le désir obsédant d'échapper à la médiocrité de leur condition. Situés dans leur milieu de vie, dans leur étonnant grégarisme, condamnés à vivre une existence aussi étriquée que l'espace qui leur a été imparti, les personnages des Chroniques du Plateau Mont-Royal et de Demain matin, Montréal m'attend expriment, dans un langage cru, les multiples visages de leur isolement. Qu'ils soient stigmatisés en raison de leurs choix de vie ou de la classe sociale à laquelle ils appartiennent, les protagonistes de Tremblay semblent avoir épousé les frontières internes de la Main et du Mont-Royal, en les intériorisant au plus profond d'eux-mêmes. Cette intériorisation a pour conséquences la détérioration de leurs rapports humains, de l'intimité des couples qu'ils forment et de leur vie familiale. Les retranchements défensifs de chacun empêchent ces individus marginalisés de faire front commun contre les problèmes quotidiens.

Ainsi, dans le monde des prostituées, travestis et homosexuels de la rue Saint-Laurent, barrière naturelle entre l'est et l'ouest de la ville, le clivage des aspirations individuelles et des limites fixées par la société et la culture dominantes s'impose. Transcendant l'opposition historique entre francophones et anglophones, la Main devient le symbole de la marginalité vécue, consentie et assumée ${ }^{2}$. Ces marginaux, comme Hosanna, la

2 Plus qu'une frontière entre l'est et l'ouest de la ville, la Main se présente aussi comme un territoire qui favorise l'anonymat, l'expression impersonnelle de la marginalité. Nos propos rejoignent ici ceux de Simon Harel : "[..] la Main introduit de l'anonymat, une quête de l'ordinaire, un refus d'appropriation territoriale. [...] le refus du nom propre ou l'attribution d'un éponyme dérisoire parce qu'offert, dans sa neutralité apparente de centre, à la multiplicité des migrations. [...] la Main à Montréal est l'expression d'un patrimoine dispersé et l'ambivalence onomastiques [...] est une façon de signifier l'attachement porté à une certaine neutralité. Trêve de combats de mots ou de 
Duchesse de Langeais, Candy-Baby, Thérèse ou Betty Bird, qui n'ont à peu près jamais exploré l'ouest de la ville, sont pour la plupart natifs du plateau Mont-Royal, lieu d'origine qu'ils ont presque tous volontairement délaissé.

Loin de s'insurger contre l'infiltration culturelle anglophone qui marque le Montréal des années quarante, ou contre la domination socio-économique des élites de l'ouest de la ville, les personnages qui fréquentent le Meat Rack, le Coconot Inn ou le Palace s'exposent volontairement à une culture qui leur est, de prime abord, étrangère. Voulant accéder à un vécu authentique et libérateur, à une nouvelle identité, tout en rêvant de goûter aux avantages de la réussite sociale, ces marginaux francophones sont liés par leur appartenance au milieu socio-économique. S'ils envahissent le nouvel environnement qu'ils découvrent avec la Main, c'est pour tenter de le façonner en fonction de leurs besoins d'expression. La fréquentation nocturne de certains clubs mal famés de la rue Saint-Laurent devient, pour eux, un idéal et une raison de vivre, une façon d'oublier leur milieu d'origine et d'échapper à leur passé. Louise Tétrault, dans Demain matin, Montréal m'attend, partage cette communauté d'expérience avec Thérèse, personnage important dans les Cbroniques du Plateau Mont-Royal. Provenant toutes deux d'un environnement familial étouffant, ces femmes, marquées par le besoin intense de défier leur destin, abandonneront le territoire qui les a vu naître pour flirter avec la réalité interdite que représente la Main. La première, récipiendaire du trophée Lucille Dumont, abandonnera son Saint-Martin natal, avec un seul objectif en tête: devenir une grande star montréalaise. Dans son esprit, Montréal apparaît comme une terre de promesses, de liberté et d'espoir:

De l'air! De l'air! De l'air! (crie-t-elle)

Donnez-moé de l'air!

Même si est polluée!

L'air sale de Montréal

Les rues sales de Montréal

C'est tout ça que j'veux connaître [...]

Si y faut être sale pour vivre, j'vivrai sale [...]

territoires, la Main permet peut-être la réunification harmonieuse d'un anonymat communément partagé». (Le voleur de parcours, Longueuil, Les Éditions du Préambule, 1989, p. 24-25). 
46

De l'air! De l'air! De l'air!

Donnez-moé de l'air même si est empoisonnée. ${ }^{3}$

La seconde, pour sa part, quittera le Plateau Mont-Royal en "crachant sur son passé ". Comme le précise le narrateur du Premier quartier de la lune, Thérèse

se contenta de cracher par terre et se sentit soulagée. Elle était un oiseau de nuit et n'aimait la rue Mont-Royal, les rues de Montréal, que corsetées de néon et presque vides, à l'heure où les commerces licites fermaient et où ceux, bars, tavernes, clubs de nuit, blind pigs qui flirtaient avec le défendu, le pas propre, le pas respectable, ouvraient leurs portes aux rêves trompeurs, faux la plupart du temps et souvent dangereux mais tellement consolants. Elle avait toujours ressenti le besoin de se consoler de vivre, avait trouvé dans la nuit un baume, une drogue, plutôt, qui engourdissait son intelligence, ses questionnements. ${ }^{4}$.

En plus d'être une lisière entre deux espaces socialement et culturellement opposés, la Main apparaît ici comme une terre d'accueil pour les parias de la société montréalaise en quête d'identité et de liberté. Considéré de l'extérieur par les citoyens des autres quartiers de la ville, le petit monde de la Main est vu comme un ghetto constitué de "soûlons", de "drogués", de " béjaunes ", d'"ignorants", de "moins que rien" qui ont profondément honte d'eux-mêmes et auxquels le personnage de Carmen, dans la pièce Sainte Carmen de la Main, voudrait bien insuffler un peu de confiance et de respect:

D'un coup qu'a l'arait envie de chanter fort pis de rire au lieu de raser les murs en se frappant la poitrine pis en braillant que c'est de sa faute si est rendue ousqu'a l'est! D'un coup qu'a l'arait envie de crier au monde: "R'gardez, chus là, me v'là " au lieu de chuchoter: “Oubliez-moé, chus laide!” D'un coup qu'a l'arait envie d'être quelqu'un qu'on écoute pis qu'on respecte au lieu d'être une trainée qu'on viole pour vingt piasses la nuit pis qu'on ignore le jour sauf de temps en temps pour dire d'elle qu'on a honte pis qu'a mérite pas de vivre! ${ }^{5}$

Si la description de cette rue de Montréal dans l'œuvre de Michel Tremblay atteste le vécu tragique d'un corps social

3 Michel Tremblay, Demain matin, Montrêal m'attend, Montréal, Leméac, 1972, p. 15.

4 Michel Tremblay, Le premier quartier de la lune, Montréal, Leméac, 1989, p. 231-232.

5 Michel Tremblay, Sainte Carmen de la Main, Montréal, Leméac, 1976, p. 64. 
marginalisé, les personnages de la Main ne ressentent pas cette exclusion sociale comme l'effet d'une sanction qui les condamne comme groupe, mais comme une atteinte à leur individualité. C'est le cas d'Hosanna, de la Duchesse de Langeais et des danseurs de Lola Lee.

Pour Hosanna, le drame qui l'afflige ne vient pas de sa situation de travesti face au monde dit "normal", mais plutôt du conflit permanent entre son désir d'être Elizabeth Taylor dans le rôle de Cléopâtre et la détermination physiologique de son sexe, qu'il rêve d'annihiler. Loin de se contenter d'un pseudonyme, aussi féminin soit-il, ce personnage est d'abord et avant tout à la recherche d'une nouvelle identité, sous la forme d'un déguisement. Lui qui ne sait parfois plus s'il doit parler de lui au masculin ou au féminin, voudrait parvenir à ressembler, jusqu'au bout des ongles, à la grande star américaine. Le dédoublement de personnalité qui contraint Hosanna, et qui tient du tragique, est la preuve de l'isolement qu'il éprouve et de l'aliénation qui en résulte. En adoptant un costume, Édouard, alias la Duchesse de Langeais, acquiert lui aussi une nouvelle personnalité. Ses vêtements féminins lui permettent de sortir du Plateau Mont-Royal, d'accéder au monde de la Main et d'en faire le royaume de son identité de parade. Le portrait de ce personnage, quelques heures à peine avant son assassinat dans un stationnement de l'est de la ville, devient plus que révélateur à cet égard:

La Duchesse faisait les cent pas devant le coconot Inn. Une louve en cage. Ou, plutôt, une louve qui faisait les cent pas à la porte de sa cage sans se décider à y rentrer. Elle avait mis le Coconut Inn au monde et le Coconut Inn ne voulait plus d'elle! [...] Fut un temps où elle pouvait réciter par cœur la liste complète de tous les habitués de tous les bars de la Main, avec leurs particularités, leurs préférences sexuelles, leurs vices cachés et ceux qu'ils arboraient fièrement. Mais les petits nouveaux qui envahissaient la rue Saint-Laurent depuis quelques années lui échappaient de plus en plus. ${ }^{6}$

Enfin, dans Demain matin, Montréal m'attend, les danseurs si "sexy" de Lola Lee sont des travestis. L'un d'eux, Butch, est en réalité une lesbienne qui travaille dans une maison de prostitution appartenant à Betty Bird. En regardant ce personnage, il est bien difficile de reconnaître l'individu qui se cache sous le costume qu'il porte.

6 Michel Tremblay, Des nouvelles d'Édouard, Montréal, Leméac, 1984, p. 22-24. 
48

Bien que la rue Saint-Laurent ne soit pas un territoire d'affrontements culturels et linguistiques, cette frontière invisible dans la ville apparaît, dans l'ouvre de Tremblay, comme une zone-tampon qui reflète un profond malaise au niveau de l'identité des marginaux de cette rue, tous francophones. Et ce malaise, on l'a vu, devient visible à travers leur déguisement. Or, se déguiser, c'est camoufler ce qui est laid, ce qu'on refuse de voir en soimême ou de montrer à la face du monde. C'est projeter une fausse image de soi et devenir quelqu'un qu'on puisse aimer aussi longtemps qu'on ne s'accepte pas avec ses défauts et ses faiblesses. C'est pourquoi le travestissement, associé à la fréquentation nocturne des clubs de nuit arborant tous des noms anglophones, se présente comme un moyen de fortune qui permet aux personnages de la Main de dépasser imparfaitement leur honte et leur impuissance ${ }^{7}$. Dans ce contexte, le déguisement devient une forme d'aliénation de l'identité francophone qui ne pourra être résolue que lorsque les marginaux admettront la vérité du jugement de Louise Tétrault: "J'ai eu le temps de me rendre compte que c'est du monde comme les autres eux autres aussi. ${ }^{8}$

Pour que les marginaux parviennent à une telle prise de conscience, encore faut-il qu'ils comprennent d'abord que, tout en structurant la vie de la Main en fonction de leur quête d'identité et de liberté, cet environnement social influence, en retour, le type de relations qu'ils ont entre eux ${ }^{9}$. Et souvent, dans un ghetto, les relations sont loin d'être libératrices. Au contraire! Par leurs interactions, les personnages de Demain matin, Montréal

7 Notons ici que les données étiologiques de la psychiatrie moderne tentent de démontrer qu'un sentiment d'humiliation, de honte ou de vulnërabilité, au plan de l'identité, serait à l'origine du travestisme. (Voir à ce sujet l'ouvrage de Pierre Lalonde et de Frédéric Grunberg, Psychiatrie clinique: approcbe contemporaine, Chicoutimi, Gaëtan Morin éditeur, 1986, p. 568-569. Selon nous, ces données étiologiques définissent très bien la situation historique des Québécois, qui vivent en terre canadienne, et peuvent expliquer le profil psychologique d'Hosanna et de la Duchesse de Langeais.

8 Michel Tremblay, Demain matin, Montréal m'attend, op. cit., p. 56.

9 Selon l'anthropologue Edward T. Hall, l'espace est le reflet de la culture. Dès lors, en fonction de ses acquis culturels, l'homme façonne un environnement qui, en retour, conditionnera ses comportements futurs: "Le rapport qui lie l'homme à la dimension culturelle se caractérise par un façonnement réciproque. L'homme est maintenant en mesure de construire de toutes pièces la totalité du monde où il vit [...] En créant ce monde, il détermine en fait lorganisme qu'il sera". (La dimension cacbée, Paris, Éditions du Seuil, 1971, p. 15). 
m'attend témoignent d'un monde régi par la frustration, l'hostilité, l'hypocrisie, l'égocentrisme et la peur. Si on rajoute à cela la très dure rivalité qui règne dans le monde des prostituées, des travestis et des homosexuels de la Main, on comprend que leur vie devient irréconciliable avec toute forme d'altruisme. Lola Lee regrette cet état de faits qu'elle dépeint à sa soeur de la façon suivante:

C'est de même dans not'métier, Louise! Tout le monde s'hait, tout le monde se bat, tout le monde s'arrache les cheveux, tout le monde s'en veut, tout le monde se fait des coups pendables, mais à la fin du compte tout le monde finit toujours par se garrocher dans les bras les uns des autres [...] tout le monde fait semblant de s'aimer... pis comme tout le monde joue... tout le monde finit par se croire. ${ }^{10}$

En fait, dans ce milieu, il est tellement difficile de vivre, de survivre ou d'arriver à quelque chose que le succès d'autrui apparaît inadmissible. L'esprit de compétition, qui pousse les individus à s'écraser mutuellement, caractérise la vie nocturne de la rue SaintLaurent.

À partir de toutes ces données, nous ne pouvons éviter d'établir un rapport entre la condition existentielle des marginaux de la Main, la dévaluation de leur réalité et l'état général de la société montréalaise considérée comme un microcosme de la société québécoise des années quarante. Dans ce contexte, le chemin parcouru par les personnages de la rue Saint-Laurent pourrait très bien préfigurer celui des Québécois vivant de cette époque, la Main illustrant un régime politique qui cache son visage d'oppression sous le dehors de la bonne entente entre francophones et anglophones, alors qu'il contribue à une véritable aliénation culturelle. Théâtre d'un monde intérieur atrophié, où il ne semble y avoir de place que pour les complexes d'infériorité, l'incommunicabilité, l'incompréhension et le manque de solidarité, caractéristiques longtemps associées aux Québécois, la Main apparaît comme un système de valeurs condamné à la stérilité.

Présentée dans sa quête d'identité, la collectivité montréalaise, dans l'cuvre de Tremblay, devient l'objet d'une dégradation croissante des valeurs sociales, qui n'est d'ailleurs pas limitée au cadre étroit de la Main. Habitant le Plateau Mont-Royal, 
Germaine Làuzon, Rose Ouimet et les autres ne connaissent pas, elles non plus, de discussion sereine. Elles se querellent continuellement, se jalousent cruellement pour se réconcilier aussitôt car, en fait, elles ne peuvent se passer les unes des autres. Il en va de même pour les enfants de la rue Fabre qui, au dire du narrateur du Premier quartier de la lune, sont le cour de cette rue: "Un nœud d'intrigues d'enfants du même âge qui s'aiment presque trop et qui ne peuvent s'empêcher de se faire autant de mal que de bien; le cœur, la source, l'origine de la vie même de la rue." 11

Comme la rue Saint-Laurent, le Plateau Mont-Royal apparaît dans les textes de Tremblay comme une entité géographique rigoureusement circonscrite ${ }^{12}$. Par la description des rues Fabre, Gilford, Papineau, Beaubien et Mont-Royal, pour ne nommer que celles-ci, l'auteur nous décrit le prolétariat montréalais à travers ses relations humaines, ses frustrations, son dépouillement et son impuissance. Ce quartier, où la marginalité se traduit par un

11 Michel Tremblay, Le premier quartier de la lune, op. cit., p. 146.

12 Mes travaux de recherche, qui sont encore au stade exploratoire, me font voir dans ces enclaves montréalaises que sont la rue Saint-Laurent et le Plateau Mont-Royal une métaphore de la situation historique des Québécois, qui vivent dans un espace réduit au minimum par des barrières culturelles et linguistiques. En empruntant cinq concepts théoriques développés par l'anthropologue Edward T. Hall, mes recherches m'amèneront à développer, d'ici peu, une approche méthodologique qui permettra de repérer et d'analyser tous les espaces qui favorisent la marginalisation des personnages présents dans le théâtre de Michel Tremblay. Ces concepts théoriques s'énoncent comme suit : la proxémie, les espaces à organisation fixe et semi-fixe, les espaces sociopètes et sociofuges. Dans un ouvrage intitulé La dimension cacbée (Paris, Seuil, 1971), Hall définit la proxémie comme l'ensemble des observations théoriques concernant l'usage que l'homme fait de l'espace en tant que produit culturel spécifique. Selon l'anthropologue américain, l'homme façonne l'espace qu'il occupe en fonction de ses acquis culturels et, en retour, l'espace ainsi façonné conditionne l'expression culturelle et relationnelle des individus. Alors que certains espaces apparaissent stables, bien délimités et associables aux structures cachées que l'homme intériorise (espace à organisation fixe), d'autres ont la propriété d'être modifiables et adaptables selon les besoins (espaces à organisation semi-fixe). Hall sépare les espaces à organisation semi-fixe en deux catégories: les espaces sociopètes (qui favorisent les contacts interpersonnels) et les espaces sociofuges (qui participent à l'isolement des individus). Ces concepts théoriques devraient me permettre d'analyser les données spatiales présentes dans l'œuvre dramatique de Tremblay, et particulièrement celles qui favorisent la marginalisation des personnages. Voir aussi l'ouvrage de Hall intitulé Le langage silencieux (Paris, Seuil, 1984). 
manque d'argent et d'espace, par le recours à une langue et à une culture dites "populaires", est présenté comme une microsociété refermée sur elle-même. Aussi, à l'exception de quelques promenades dans l'ouest de la ville, sans se rendre cependant plus loin que chez Eaton, les personnages de Tremblay ne sortent jamais du périmètre que constituent les limites du Plateau. On peut le constater dans plusieurs passages des Cbroniques du Plateau Mont-Royal et, plus particulièrement, dans les toutes premières pages de La grosse femme d'à côté est enceinte:

Mercédès avait rencontré Béatrice dans le tramway 52 qui partait du petit terminus au coin'de Mont-Royal et Fullum pour descendre jusqu'à Atwater et Sainte-Catherine, en passant par la rue Saint-Laurent. C'était la plus longue ride en ville et les ménagères du Plateau Mont-Royal en profitaient largement. [...] Tant que le tramway longeait la rue Mont-Royal, elles étaient chez elles... Mais quand le tramway tournait dans la rue SaintLaurent vers le sud [...] elles [...] se renfonçaient dans leurs bancs de paille tressée [...] Aussitôt le coin des rues SaintLaurent et Sainte-Catherine tourné vers l'ouest, la liesse reprenait de plus belle et remplissait le tramway de cris sonores et de rires pleins et sentis [...] Les dernières descendaient chez Eaton au coin d'University. Jamais personne du groupe n'allait plus loin que chez Eaton. À l'ouest de ce grand magasin c'était le grand inconnu: l'anglais, l'argent [...]. ${ }^{13}$

À la fin de ce passage, le narrateur précise même: "[...] jamais personne n'allait jusqu'à Saint-Henri et jamais personne de SaintHenri ne venait jusqu'au Plateau Mont-Royal" ${ }^{14}$. Limités aux frontières de cette enclave dans la ville ${ }^{15}$, à cet univers de ruelles encombrées, de fonds de cours remplies de cordes à linge et de fenêtres ouvertes sur celles de tout le monde, les habitants du Plateau Mont-Royal semblent vivre dans la crainte de l'inconnu et de l'étranger. Dans son premier récit autobiographique, intitulé Les vues animées, Michel Tremblay traduit bien cette réalité sociale:

Nous étions arrivés au cinéma La Scala, au coin de Papineau et Beaubien, par le tramway 45 , tous excités parce que nous

13 Michel Tremblay, La grosse femme d'à côté est enceinte, Montréal, Leméac, 1986 , p. 22-25.

14 Ibid., p. 25.

15 Ginette Michaud associe le Plateau Mont-Royal, illustré dans l'œeuvre de Tremblay, à un "îlot dans l'île" de Montréal. ("Mille plateaux: topographie et typographie d'un quartier", Voix et images, $\mathrm{n}^{\circ} 42$, printemps 1989, p. 467). 
52

allions rarement dans le nord de la ville, la plupart des incursions de nos familles respectives en dehors du Plateau MontRoyal se faisant dans ce que nous appelions le bas de la ville, autrement dit la rue Sainte-Catherine. Au nord de Laurier, Montréal était pour mes amis et moi une terra incognita qui appartenait aux étrangers mais pas aux Anglais, aux autres, les Italiens, sauf peut-être Rosemont où quelque-uns d'entre nous avaient de la parenté et où on disait que ça parlait juste français; moi $[\ldots]$ je ne m'y étais même jamais aventuré et je [...] m'attendais à ce que tout change passé le viaduc, cette barrière évidente entre les Italiens et nous, érigée pour nous empêcher de nous mélanger [...]. ${ }^{16}$

Si un simple viaduc peut devenir une barrière de sécurité dans la ville, au plan culturel et linguistique, le Mont-Royal, pour sa part, apparaît comme un mur de protection qui isole, comme deux territoires distincts, les quartiers pauvres des quartiers riches. Dans le récit Les vues animées, l'auteur écrit :

[Pour ma mère], Outremont était une terre inconnue, un pays défendu où les riches se promenaient toute la journée en se saluant et en se demandant des nouvelles de leurs enfants qui étudiaient en Europe [...] pour moi, c'était un mot nouveau que je trouvais très beau, très musical,[...] je m'étais dit, tiens, y'a quequ'chose de l'autre côté de la montagne... Je voyais tous les jours la montagne au bout de la rue Mont-Royal, comme un mur qui clôturait le monde, une fin naturelle à ce qu'il m'était permis de connaitre et voilà que j'apprenais que quelque chose, un quartier, une ville, continuait Montréal au-delà de ce je croyais être la fin du monde. Quand j'avais demandé à ma mère c'est quoi, ça, exactement, Outremont, elle m'avait répondu: "C'est une place oùsque le monde comme nous autres font le ménage. "Et la barrière naturelle s'était relevée toute seule. ${ }^{17}$

Miroir fidèle du milieu populaire québécois, véritable société fermée, repliée sur elle-même dans une sorte de réflexe d'autodéfense, le Plateau Mont-Royal des années quarante devient, dans les cinq volumes des Chroniques de Tremblay, un monde marqué par la faillite des relations humaines et de la communication. Cette faillite se manifeste, en particulier, par l'isolement des personnages qui deviennent prisonniers de leur entourage familial.

16 Michel Tremblay, Les vues animêes, Montréal, Leméac, 1990, p. 67-68.

17 Ibid., p. 26. 
Épousant la fonction isolante des frontières du Plateau, rigoureusement circonscrites, les familles du ghetto se présentent comme des entités fermées particulièrement propices à l'éclosion de l'incommunicabilité et de la frustration. Par exemple, si Léopold et Marie-Louise, dans $\dot{A}$ toi pour toujours, ta Marie-Lou ${ }^{18}$, n'ont jamais pu se parler autrement qu'en s'invectivant, cherchant à se détruire mutuellement, c'est qu'ils sont profondément frustrés. Alors que le mari s'abêtit au travail sans pouvoir assurer une vie convenable à sa famille, l'épouse, elle, se sent dépassée par les problèmes d'ordre matériel (manque d'argent, étroitesse du logement) et par l'annonce d'une nouvelle grossesse. Cette frustration atteint son paroxysme au moment où, écœuré par cette lutte quotidienne dont l'objectif est de survivre plutôt que de vivre, Léopold ne trouve qu'une issue, le suicide, où il entraîne avec lui sa femme et son plus jeune enfant. Ce faisant, ces personnages désertent un environnement étroit et une société qui ne leur permettent pas de faire face à leurs responsabilités. On pourrait dire sensiblement la même chose des relations qu'entretiennent ensemble Victoire et Albertine ou Albertine et Thérèse, interactions marquées au sceau de l'incommunicabilité et de la frustration.

Les femmes du Plateau Mont-Royal partagent le lot affligeant des grossesses non désirées, des époux absents ou alcooliques et chômeurs, lorsqu'ils sont présents au foyer, des enfants ingrats et difficiles. Elles restent captives des tâches quotidiennes crétinisantes et s'usent à lutter contre un monde hostile, l'énergie nécessaire pour changer quoi que ce soit à leur vie leur faisant défaut. Une sorte d'aliénation progressive et contagieuse leur parait inéluctable. La rue Mont-Royal ou la rue Sainte-Catherine se présente alors, le samedi après-midi, comme une drogue libératrice qui donne à ces femmes le sentiment éphémère d'échapper à leur lamentable destin. L'évidence de ce sentiment d'évasion associé à la rue Sainte-Catherine s'impose avec force dans La duchesse et le roturier:

Là rue Sainte-Catherine venait d'être dégagée de sa couche de neige rapidement transformée en boue et commençait à se remplir de sa faune habituelle du samedi après-midi. [...] Effectivement, chaque samedi après-midi, les femmes de l'est de Montréal, leur progéniture accrochée à leur manteau [...] enva- 
54

hissaient la Catherine sans un sou en poche mais les yeux grands et les bras tâteux. Elles touchaient à tout, surtout quand elles faisaient bande avec des amies rencontrées par hasard ou non, parlaient fort et franc, riant d'un gros rire libérateur quand elles tombaient sur quelque chose quelles trouvaient laid ou qu'elles ne comprenaient pas. ${ }^{19}$

Prisonniers, comme leurs parents, d'une réalité familiale aliénante et d'un environnement restreint à l'intérieur duquel on retrouve des maisons blotties les unes contres les autres, derrière quelques mètres de pelouse, les enfants du Plateau Mont-Royal tentent de se libérer de ce monde en faillite en s'inventant des pays à conquérir. Mais comme on peut le noter dans La duchesse et le roturier, ces jeux d'enfants sont de piètres outils de libération:

Marcel et son cousin se tenaient éloignés des piquets de métal peints en vert ou en marron qui dépassaient à peine de quelques pouces de la neige, dessinant de longs rectangles que les enfants du voisinage appelaient des "pays" ou des "terres" korsqu'ils jouaient à la guerre et qu'ils devaient envahir le fief de leurs adversaires pour marquer des points. Ils disaient: "Le parterre des Jodoin, c'est mon pays; celui des Amyot, c'est le tien!" Et parce qu'ils avaient peur des piquets, les guerres se faisaient toutes en dehors des "pays" et des "terres"; ils rendaient les armes ou triomphaient sans jamais jouir des territoires conquis ou se sentir frustrés de ceux qu'ils perdaient parce qu'ils ne sien approchaient jamais. ${ }^{20}$

Parmi tous les enfants du Plateau, Marcel, victime de sa schizophrénie, est le seul personnage à pouvoir véritablement échapper aux contraintes de ce milieu. Comme le précise le narrateur de $L a$ duchesse et le roturier, Marcel "avait une grande facilité à s'échapper de la maison, de la rue Fabre, du Plateau Mont-Royal, pour se réfugier dans quelque pays de son invention où les enfants ne faisaient pas de sieste et où les tranches de pain n'avaient pas de croûte" 21 . Cet exemple nous invite à comprendre que seule la maladie mentale permet vraiment aux personnages de Tremblay d'échapper à un monde qui refuse à tous une vie empreinte de dignité réelle. Il serait très difficile de dire si la schizophrénie de Marcel est héréditaire ou conditionnée par l'environnement social et familial. Néanmoins, si elle est le refuge du plus marginal des

19 Michel Tremblay, La duchesse et le roturier, Montréal, Leméac, 1982, p. 195.

20 Ibid., p. 171-172.

21 Ibid., p. 210. 
marginaux, des plus faibles ou des plus désarmés, la folie apparaît, dans les textes de Tremblay, comme le symptôme d'une société en voie de dissolution, et dont les membres, privés de cadres, de soutien et d'espoir, sont condamnés à la désertion, du moins mentalement, puisqu'ils sont impuissants à changer leur condition.

En somme, tout comme la Main et le Plateau Mont-Royal, qui illustrent de manière probante le repli aliénant sur soi-même, la famille dysfonctionnelle reflète, dans l'œuvre de Michel Tremblay, la société montréalaise des années quarante. Elle renferme en son sein, au moins virtuellement, l'ensemble des rapports discordants de cette société aux plans social, politique et culturel. De nombreux facteurs ont annihilé l'unité de la famille québécoise, ont fait éclater ce réseau de socialisation primaire, la privant de ses fonctions d'encadrement et de soutien mutuel; ces facteurs ont entamé les liens affectifs et économiques de ces familles pour ne laisser subsister qu'un réflexe individuel d'auto-conservation: l'incapacité de maîtriser la réalité quotidienne, liée à une perception défaitiste du réel ; une communication insatisfaisante ou destructrice; le besoin individuel de s'en sortir ne s'affirmant que dans l'échec des rapports interpersonnels, les rivalités, l'hostilité et l'agressivité. 